\title{
Identification of Lost Children using Face Aging with Conditional GAN
}

\author{
Amisha Pupala ${ }^{1,3, *}$, Samruddhi Mokal ${ }^{2, * *}$, Neha Pandit ${ }^{3, * * *}$, and Smita Bharne ${ }^{4, * * * *}$ \\ ${ }^{1}$ Ramrao Adik Institute of Technology, Navi Mumbai, India
}

\begin{abstract}
Face recognition technology is a big area that consists of the many features in it but it also comes with some of the factors which affect this technology, one of the factors is Face aging which makes face recognition more difficult. As in India, a large number of children go missing every year. Also just using a photograph is not enough for the process to proceed smoothly and it results in a huge percentage of the missing child cases remain untraced. This paper presents a novel use of face recognition with face aging to overcome the limitation of existing systems. The proposed system has a portal where the public can upload an image of a suspected child and also have a feature where searching for any lost child is possible. The proposed system has mainly concentrated on an Age Conditional generative adversarial network (C-GAN) algorithm for face aging and the FaceNet algorithm for face feature extraction and face recognition.
\end{abstract}

\section{Introduction}

Children represent a significant percentage of the total population of India. Unfortunately, many children go missing every year because of numerous reasons like kidnapping, trafficked children and lost children, run-away children, and abduction. According To the National Crime Record Bureau 2019 report, the number of missing children has increased by 8.9 percent since 2019 giving a total of 73,137 children reported missing last year. Many NGOs claim that the actual number of missing children cases is much more than the reported number. A child lost in one location can be found in some other location. Even if the child is found it gets difficult to recognize him/her. Face recognition is thus the most promising biometric technology for recognizing and finding missing children. But if the child is found after many years from the date when he/she is lost the state-of-art face recognition systems fall short to identify children from the photograph because a child's face undergoes many temporal variations due to aging like a change in skin texture, change in the shape of the face, color, weight, facial hair growth, etc. Hence, as the time difference between a true mate image and probe image gets larger, the performance of the face recognition algorithm decreases, and thus the search gets harder. Thus the primary goal of the proposed system is to generate an age-progressed image of the child rather than enhancing the face recognition performance. Thus this system enhances the ability of face matching algorithms which will be used to identify and locate children who are lost at a young age by aging face features to reunite them back with their families. Developed system will be helpful to the po-

\footnotetext{
*e-mail: amishapupala20@gmail.com

**e-mail: samruddhimokal10@gmail.com

***e-mail: panditneha124@gmail.com

****e-mail: smita.bharne@rait.ac.in
}

lice and higher authorities for tracking down missing children quickly.

\section{Literature Survey}

In 2018, S. Chandran publish a paper [1] in which authors have used CNN and VGG face technologies for the identification of missing children. The faces of children are detected and features are extracted by using CNN. CNN is an important tool that works appropriately with image data. Blocks of Relu pooling layers, convolutional, and fully connected layers are composed in CNNs or ConvNets. For classification, authors have used the SVM Classifier. But these technologies slow the process of classification and extraction of faces from images.

In 2020, a Face-to-face matching method is based on IPEbased blind computing which is used to restrict access to photos [2]. Using Multitask FaceNet Model, the system can safely run and provides privacy for both the unsure and missing children parts. To provide a more effective face recognition module, the output of images with encryption schemes needs to be improved.

In [3], VGG16, ResNet50, and Mobile-FaceNet these three Convolutional neural network algorithms were used. From which Mobile-FaceNet has the smallest model size and VGG16 has the largest model in size.

In [4] authors have used DeepId and modified Inception structures which contain different types such as DeepId2, DeepId3 modules which give the best performance with higher accuracy when there is very little difference between a deep module and a wide module. however, the mutual information goes down with additional branches such as DeepId3 in a wide module.

Face aging with Age-cGAN to generate artificial images within different age categories [5]. The face recognition method used by this system is the Identity preserving la- 
tent vector optimization method used to conserve the original photo's identity while face aging which can be improved by combining Pixelwize and Identity-Preserving methods into one singular method.

\section{Proposed Work}

It is known that the performance of the face recognition algorithm is compromised due to aging. It is still a problem to consider the aging parameter under consideration for face recognition as most of the algorithms fail to detect the face of the child after a few years. A child's face undergoes various changes including facial hair, weight, the texture of the skin, shape, and size of the face, etc. Several studies were performed to analyze the effects of face aging on the performance of the face recognition algorithms. Two conclusions can be drawn from these studies:

1. The performance of traditional face recognition algorithms decreases with an increase in time-lapse.

2. Performance of face recognition and detection algorithms decreases more in the case of younger individuals as compared to older individuals.

Hence it is important to consider the age progression of the face for enhancing the performance of the face recognition algorithm, especially when enrolled at a young age. A. In particular, our contributions are as follows:

- A portal for finding missing children that compare the image of the missing child with the images of the already registered missing children.

- To take into consideration many kinds of variations in face images which are taken under uncontrolled conditions such as change of expression, illumination, pose, change in lighting condition, presence of noise, and blurred image.

- Using Age Conditional Generative Adversarial Network to reconstruct synthetic images of the required. Using methodology like Facenet and C-Gan the process of recognition gets easier which will help the authorities to find the missing children quickly and solve many cases which have been untraceable because of the above issues.

Thus the proposed system presents a novel deep learning methodology to find the lost children using the photo of the children currently available using face recognition. Whenever anyone finds any suspicious child on the road he/she can click the photograph of the child and upload it into the common portal with landmarks and remarks. The public has to upload the photograph of the missing child into the portal. This photo will automatically be compared with the already saved images in the repository. If no matching record is found then the image will be age-progressed and again the age-progressed image will be compared with the registered photographs. For this, a deep learning model Aging cGAN is used. And the face recognition is done using FaceNet. Thus the proposed system outperforms the earlier methods in face recognition. As mentioned earlier, the face aging model is based on cGAN, a model for the synthesis and generation of human faces of the required age category. Hence the purpose of cGAN network is to generate the image of a child at different ages based on the input image and target age. The structure of the model consists of three parts: encoder, age condition, and decoder. The encoder is used for feature extraction of the input face image. Proposed system is using three groups of convolution layers and nine residual blocks. Each convolution layer consists of a ReLU non-linearity layer, spatial batch normalization layer stride, and a convolution layer. After this, it will give a 56 feature map of the image. Since there is a huge range of images of a large number of age groups in the dataset system is dividing the image into $\mathrm{y}$ number of groups which is represented as a one-hot age vector. Each block represents a specific age group. The task of the decoder is to convert the face to generate an image of the required age group based on the features.

\section{Proposed Methodology}

A. Age C-GAN Algorithm Conditional Generative Adversarial Network is conditioned on extra information and is based on the idea of Vanilla GAN which allows to control the output of the generator network. This system have to give extra information $\mathrm{y}$ as an additional layer to the generator. As compared to vanilla GAN, In vanilla GANs no one can control the category of the retrieved images as it can learn only one category, however, in CGAN can generate images of the specific category using condition $y$. $\mathrm{y}$ can be any data depending on the application for which the model for like integer data or class label has been built. Thus CGAN can be used to generate models with different categories and conditions. The Face Aging C-GAN has four networks: Encoder: An encoder is a deep convolutional neural network used to generate the latent vector of images. It is used to learn the inverse mapping of input image and age condition with the latent vector $\mathrm{Z}$.

- It generates a latent vector of 100 dimensions of the input face images of dimension $(64,64,3)$. There are 2 dense layers and 4 convolution blocks.

- All the convolution blocks except the first layer have a convolution layer which is followed by a batch normalization layer and then an activation function.

Generator Network: It is a deep convolutional network that takes a face image and condition vector and tries to generate realistic images of the face.

- It is a CNN having upsampling, convolutional, and dense layers which take a condition vector which is additional information added to the network which is age, and a latent vector to generate a realistic image of a dimension of $(64,64,3)$. The condition vector is the additional information that is provided to the network. For the Age-cGAN, this will be the age.

Discriminator Network: It is CNN that is used to discriminate between the real image and the fake image. It contains several convolutional blocks which contain a batch normalization layer, convolutional layer, and an activation function. 
FaceNet: It is a face recognition model that learns the difference between the generated image $\mathrm{x}$ ' and the original image $\mathrm{x}$ (input image). It is used to compare the person's identity in the image. The proposed system is using a ResNet2

For FaceNet Inception ResNet, ResNet-50, or pretrained Inception can be used. This system using the ResNet 2 model without fully connected layers. FaceNet is used to identify the person's identity in the given input image. The extracted embeddings for the original input image and the reconstructed image can be found by calculating the Euclidean distance between the embeddings.

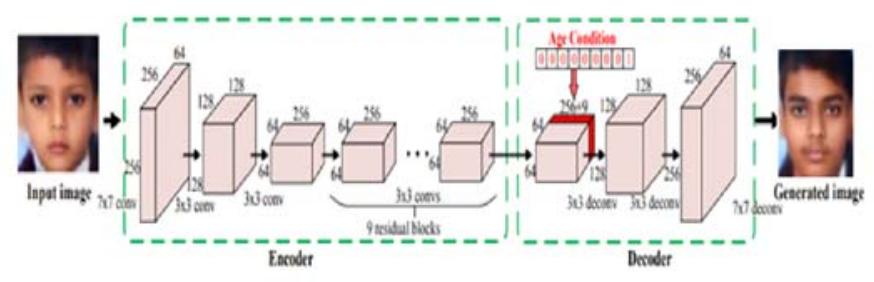

Figure 1. Layers used in Aging cGAN

\section{Stages of Age-cGAN:}

1. Age-cGAN has four networks and it is trained in three stages: Conditional GAN training: In this stage, The System trained the generator network which generates the blurred images of the face after training and the discriminator network.

2. Initial latent vector approximation: In this stage,The System trained the encoder network to approximate the latent vector using both the generated images and the real images which generates the latent vector from learned distribution.

3. Latent vector optimization: In this stage,The System optimized both the encoder and the generator network.

\subsection{FaceNet}

This System uses FaceNet for feature extraction and face recognition.The System first passed all the images through Multi-Task Cascaded Convolutional Neural Network (MTCNN) for finding and extracting faces from the images. Then system used FaceNet for extracting highquality features from children's faces which been used to train a face identification system. It uses Convolution layers to learn embeddings from the face directly which are used for face verification and face recognition. These embeddings are of 128 dimensions and insert them into feature space such that the squared distance between faces of the same person is less whereas the squared distance between images to two different people is large.

\section{Dataset}

A. Dataset for Face Recognition

This system used two separate datasets for the training of

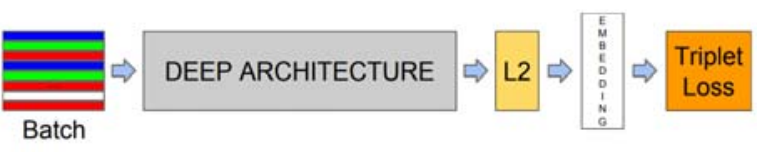

Figure 2. Model architecture of FaceNet.

face recognition and the Age Progression model. For face recognition, dataset been created by collecting images of children from various sources. In a proposed system there is a directory structure where each child to be recognized has a dedicated directory with their images saved in it. The photos in the directory provide a range of orientation, sizes, lighting conditions. If an image contains multiple faces then the face having the highest probability will be considered for further steps. The system uses this dataset as the basis for the classifier which is trained on a training dataset only.

\section{B. IMDB Wiki-Crop Dataset}

For training the Age progression model,The System used the IMDB WIKI-crop dataset which is the largest publicly available dataset of human faces. It contains about 400 thousand images where each image is labeled in the format gender, age, the name with all the metadata information like dob, the year when the photo was taken, gender, face location, face, and secondary face score, etc in the .mat file. The dataset contains images of all the age categories from 0 to 100 years of age which is not required for the model. So the system have to filtered the dataset and considered the images of children in the age group from 0-15 years of age and have deleted the rest of the images. The dataset contains images with huge variations in facial expression, illumination, resolution, occlusion, pose, etc. The dataset also provides the corresponding landmarks. This dataset can be used for a variety of tasks including age estimation, age progression, landmark localization, progression/ regression, face detection, etc.

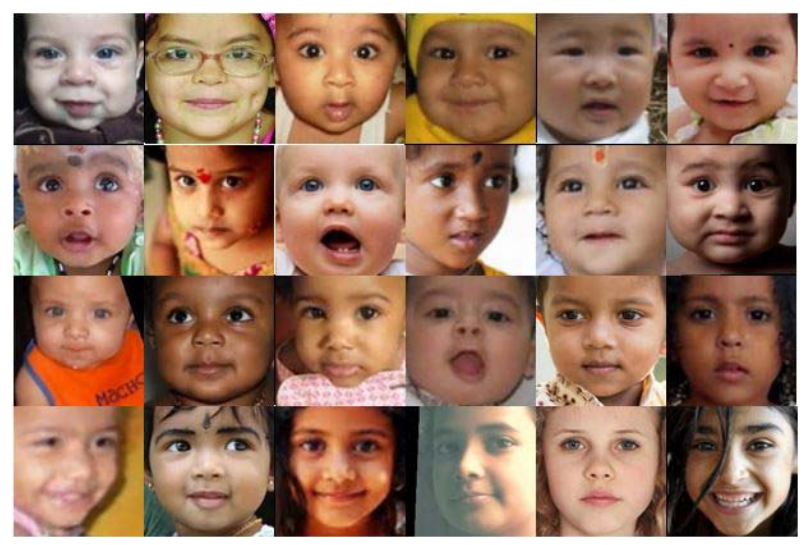

Figure 3. Wiki Sample Dataset

\section{System Design}

The proposed system consists of a portal where the public can store the details with a photograph of the missing 


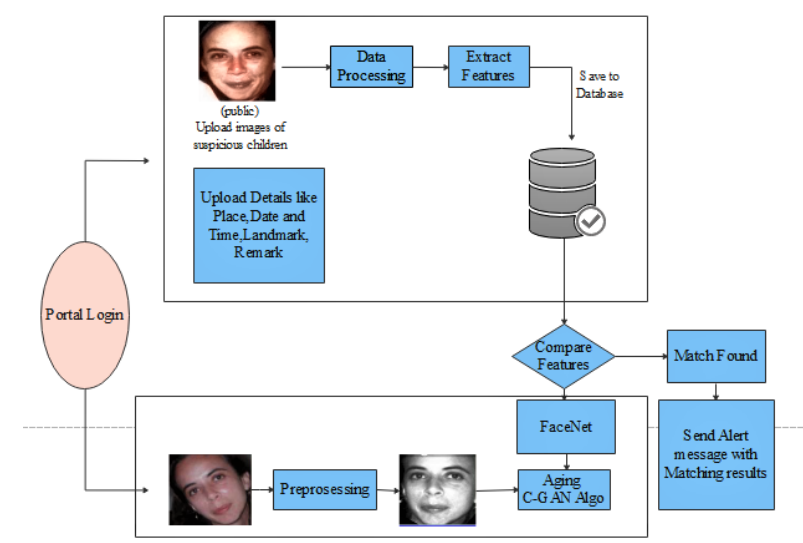

Figure 4. System design for lost child idetification using FaceNet and C-GAN.

child. The public here refers to the parent of the missing child or the police officer. By using the photograph the public can find the matching children from the already stored data. The system will prompt the most matching cases after applying a face recognition algorithm to the uploaded image. Once the matching is done then the concerned person will get the details of the child. The proposed model is divided into various phases. First, whenever the public finds any missing or suspicious child they can upload the photograph of the child with the details like name of the child, age, place where the child was found, date and time when the child was found and remarks like what clothes the child was wearing when he was spotted or any information the child might have told to the person which can be helpful to find the child. This uploaded image goes through an image pre-processing phase. The input to this is the raw image that is uploaded by the public and the output of this phase is an aligned frontal face image. The main purpose of this step is to align all the face images based on eye coordinates such that all the images are aligned with the same standard size.This system used a Multi-task Cascaded Convolutional Networks (MTCNN) model which is a framework used for face alignment and face detection which consists of three stages of convolutional networks that can detect landmark location such as eyes, nose, and mouth and can recognize face very efficiently. The preprocessing step includes removing noise from the image, straightening the image, and detecting and cropping the face from the whole image. The extracted face features will be stored in the database for further processing. To search for a missing child parent of the child needs to upload a photograph of the child then this image will go through the face recognition algorithm after the image pre-processing and feature extraction step.If any matching photo is found in the database then the image of the child will be displayed on the screen.

If no matching image is found then the uploaded image goes through the Age CGAN algorithm. The GAN algorithm is used to generate a new age-progressed image with the same statistics as the training images. As the child may be spotted after many years, the face of the child changes from the last known photograph taken before the child had

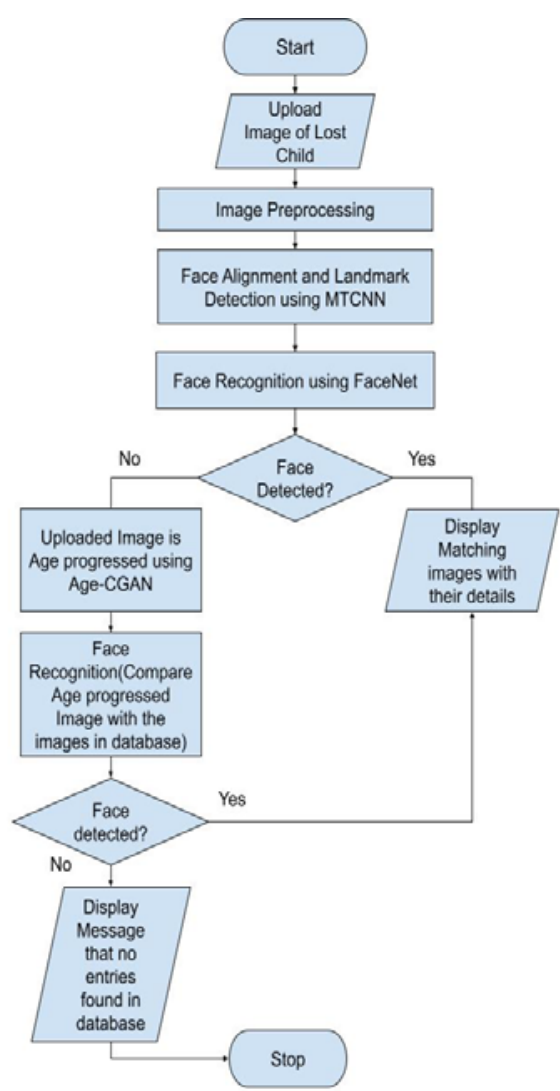

Figure 5. Proposed Flow of the System.

gone missing. So this model will be used to synthesize the child's face to produce an age-progressed facial image of the child. So basically this model will be used to synthesize child faces to the required age category. This face synthesized and age-progressed image goes through the FaceNet algorithm for feature extraction and face recognition. The main distinction between FaceNet and other face recognition algorithms is that FaceNet does not use any bottleneck layer for verification and recognition tasks but rather learns the mapping directly from images and creates embedding. The FaceNet is used to automatically compare the age-progressed photo of the missing child with the already present images in the database and if the photo matches with any of the photos present in the database then it will be displayed on the screen along with details like name, location and time where the child was found.

\section{Result and Analysis}

The performance of the face recognition algorithm was evaluated on a child dataset before and after age progressing the child images. The Age cGAN model was trained on the IMDB Wiki- Crop dataset. The IMDB Wiki-Crop Dataset contains more than 400k images with system The system filtered that dataset and have just used images from the age group 0-15 years to train the model.

Based on their experiments, the following conclusions are derived: 


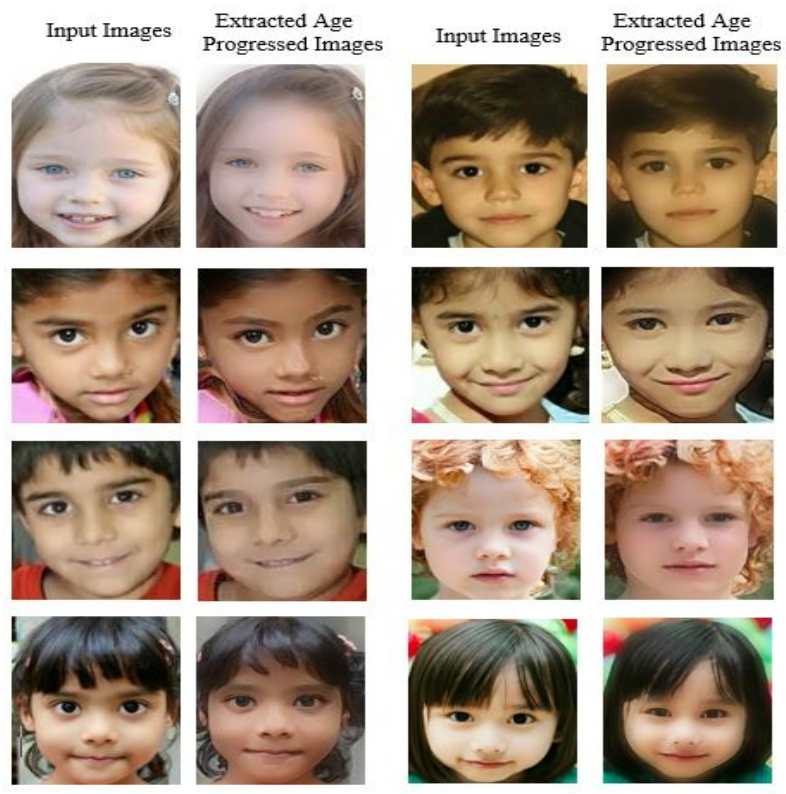

Figure 6. Retrieved Age progressed images of the proposed system

- The accuracy of the face recognition algorithm with the images of the same age category is train Accuracy: 87.38\% and test Accuracy: 81.07\%.

- The Accuracy of face recognition algorithm with images of different age categories: $44.76 \%$.

- The Accuracy of the FaceNet algorithm with images of different age categories after age progression images using Age-cGAN algorithm: 47.32\%.

Based on the experiments, the following conclusions are derived:

- The model correctly recognizes the misaligned faces and images clicked in an uncontrolled environment like the change of expression, illumination, pose, change in lighting condition, and presence of noise.

- The average decrease in the performance of face recognition is more than $40 \%$ due to age variation in the faces of the children

- Intra-person variations like expression and pose are degrading the performance of the system is similar to face aging. Also, large self-occlusion, blur, and misaligned faces are slightly degrading the performance of the model.

1) Limitations:

- Training of the deep learning model which requires a large dataset remains a challenge when training with a small dataset hence accuracy is compromised.

- The performance of the face recognition model heavily depends on the accuracy of age-progressed images which are obtained.

- Due to the long duration of code execution and heaviness of the model proposed system have trained the model for 300 epochs due to which the performance of the model is compromised.
2) Suggested Solutions:

- As face aging depends on various other As face aging depends on various other factors other than aging, it is required to consider all the other factors like having a single face aging database with correct demographic details and a lot of images of the child of various age groups is the need of the hour for better accuracy of the face aging model.

- The model age progresses the images of the child to just one age group, this can further be improved in the future by using a bigger dataset and categorizing the images in the dataset in various required age groups.

- The Age progression model should be trained for at least 500 epochs for getting better accuracy for ageprogressed images.

Table 1 Identification accuracy for previous work with and without the proposed feature aging module.

\begin{tabular}{|c|c|c|c|c|}
\hline Paper & $\begin{array}{l}\text { Method } \\
\text { Used }\end{array}$ & Dataset & $\begin{array}{l}\text { Without } \\
\text { Aging } \\
\text { Module }\end{array}$ & $\begin{array}{c}\text { With } \\
\text { Aging } \\
\text { Module }\end{array}$ \\
\hline $\begin{array}{l}\text { FaceNet: } \\
\text { A unified } \\
\text { embedding } \\
\text { for } \\
\text { face } \\
\text { recognition } \\
\text { and } \\
\text { clustering[6] }\end{array}$ & FaceNet & CFA & 38.16 & 55.30 \\
\hline $\begin{array}{l}\text { CosFace: } \\
\text { Large } \\
\text { margin } \\
\text { cosine loss } \\
\text { for deep } \\
\text { face } \\
\text { recognition[7] }\end{array}$ & CosFace & ITWCC & 60.72 & 66.12 \\
\hline $\begin{array}{l}\text { A Review } \\
\text { of Face } \\
\text { Recognition } \\
\text { against } \\
\text { Longitudinal } \\
\text { Child } \\
\text { Faces[8] }\end{array}$ & Cognitec & ITWCC & - & 41.1 \\
\hline Li et al & MFDA & FG-NET & - & 47.50 \\
\hline $\begin{array}{l}\text { Face aging } \\
\text { conditional } \\
\text { generative } \\
\text { adversarial } \\
\text { networks[9] }\end{array}$ & C-GAN & MNIST & - & 47.5 \\
\hline
\end{tabular}

Table 2 Model Accuracy

\begin{tabular}{|c|c|c|}
\hline Model & Age Category & Accuracy \\
\hline Face Recognition & Same & $81.07 \%$ \\
\hline Face Recognition & Different & $44.76 \%$ \\
\hline Age Progressed & Different & $47.32 \%$ \\
\hline
\end{tabular}

\section{Conclusion}

In this work, a proposed system is used for an ageinvariant face recognition system for finding missing children. The system reduces the manual work of scanning 


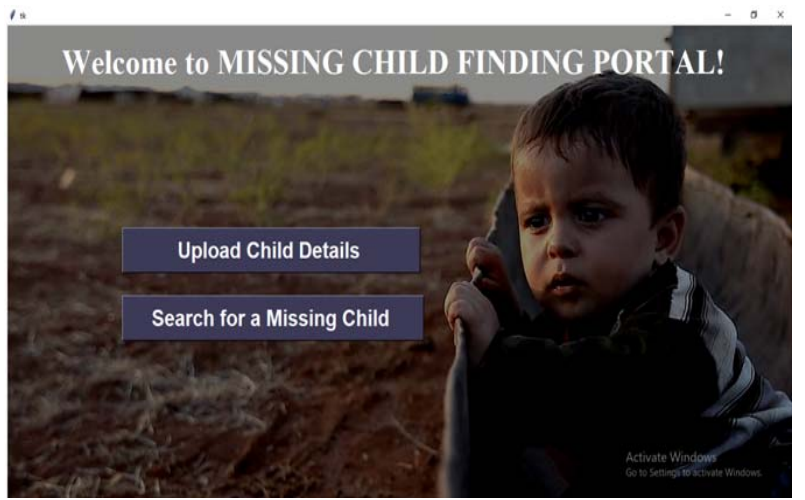

Figure 7. Welcome page of a missing child finding portal.

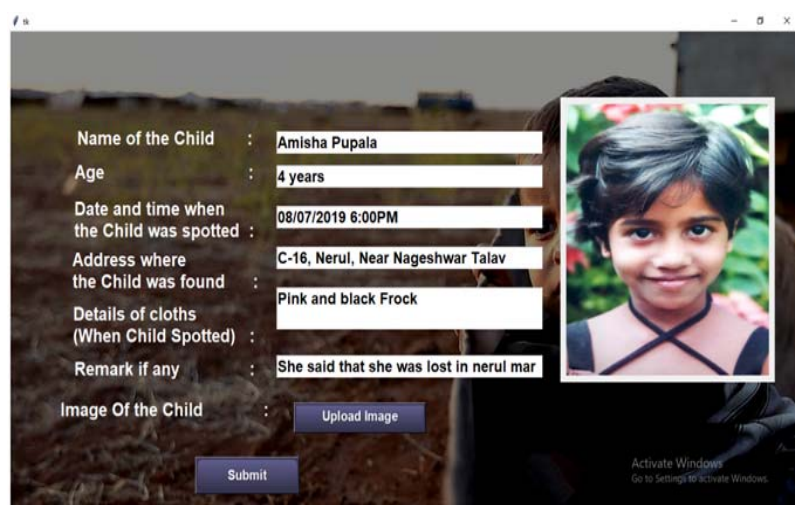

Figure 8. Page where the public can upload details of a suspicious or lost child.

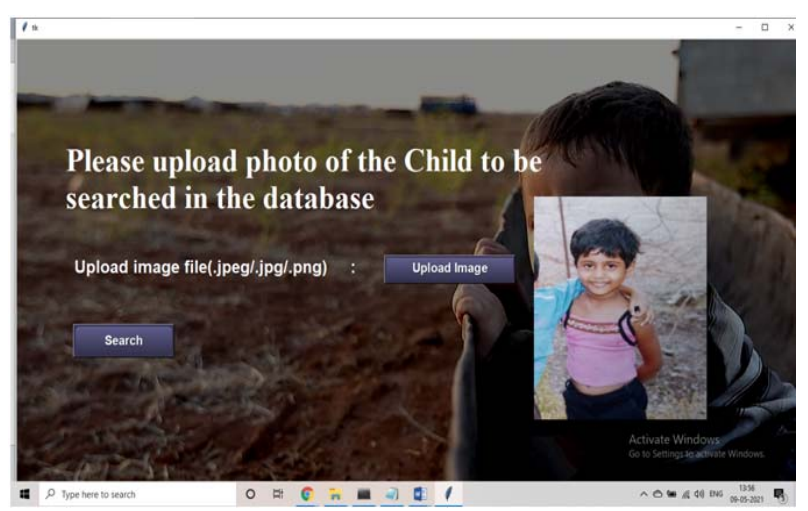

Figure 9. Page to search for a lost child using his/her photograph.

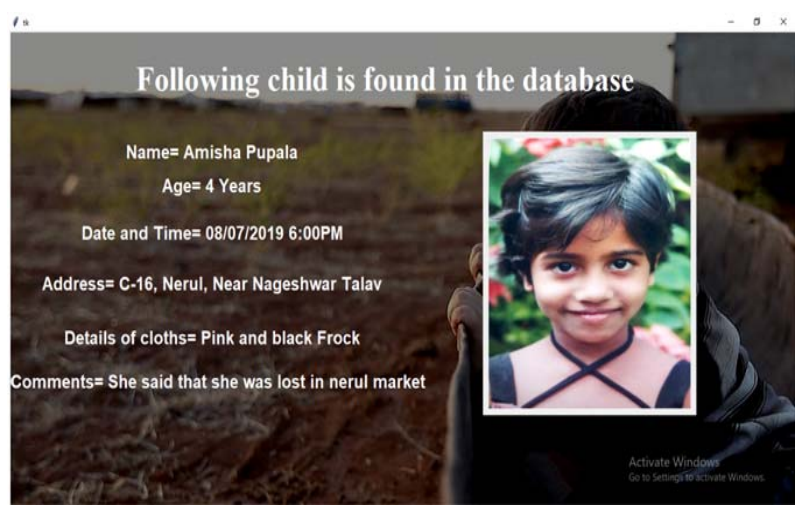

Figure 10. Details of the child found in the database through all the photographs of the lost children to find a match. The System has tried to solve the problem of age variation by age progressing the images of the child using the Aging cGAN deep learning algorithm. The model improves the performance of the FaceNet model for face recognition from $44.76 \%$ to $47.32 \%$. These results suggest that age progression of the faces of the children before face recognition enhances the ability of the system to identify the lost children. This will be beneficial for solving cases of untraced missing children and thus will help to reduce the increasing number of missing child cases.

\section{References}

[1] S. Chandran, Pournami Balakrishnan, Byju Rajasekharan, Deepak N Nishakumari, K Devanand, P M Sasi, P. (2018). "Missing Child Identification System Using Deep Learning and Multiclass SVM".

[2] 2. Xin Jin, Shiming Ge, Chenggen Song, Xiaodong Li, Jicheng Lei, Chuanqiang Wu, and Haoyang $\mathrm{Yu}$ "Double-Blinded Finder: A Two- SidePrivacyPreserving Approach for Finding Missing Children"(2020).

[3] Shun Lei Myat Oo, Aung Nway Oo University of Information Technology, Yangon, Myanmar " Child Face Recognition with Deep Learning”(2019).

[4] Chang Shu School of Communication and Information Engineering, University of Electronic Science and Technology of China Chengdu, China "Optimizing deep neural network structure for face recognition" (2017).

[5] Grigory Antipov, Moez Baccouche, Jean-Luc Dugelay "Face Aging with Conditional Generative Adversarial Networks" (2017) arXiv:1702.01983v2.

[6] Florian Schroff, Dmitry Kalenichenko, and James Philbin. Facenet: A unified embedding for face recognition and clustering. In CVPR, pages 815-823, 2015.

[7] Hao Wang, Yitong Wang, Zheng Zhou, Xing Ji, Dihong Gong, Jingchao Zhou, Zhifeng Li, and Wei Liu. Cosface: Large margin cosine loss for deep face recognition. In CVPR, 2018

[8] Karl Ricanek Jr., Ph.D. Senior Member IEEE, Shivani Bhardwaj, Michael Sodomsky A Review of Face Recognition against Longitudinal Child Faces

[9] FACE AGING WITH CONDITIONAL GENERATIVE ADVERSARIAL NETWORKS GrigoryAntipov, MoezBaccouche, Jean-Luc Dugelay 